\title{
Deepening Visitor Engagement with Museum Exhibits through Hand-crafted Visual Markers
}

\author{
Susan Ali, Boriana Koleva, Ben Bedwell, Steve Benford \\ School of Computer Science, University of Nottingham \\ Nottingham, UK \\ \{firstname.lastname\}@nottingham.ac.uk
}

\begin{abstract}
Visual markers, in particular QR codes, have become widely adopted in museums to enable low cost interactive applications. However, visitors often do not engage with them. In this paper we explore the application of visual makers that can be designed to be meaningful and that can be created by visitors themselves. We study both the use of these markers as labels for portraits that link to audio recordings and as a mechanism for visitors to contribute their own reflections to the exhibition by drawing a marker and linking an audio comment. Our findings show visitors appreciated the use of the aesthetic markers and engaged with them at three levels - physical placement, aesthetic content and digital content. We suggest that these different levels need to be considered in the design of future visiting systems, which make use of such markers, to ensure they are mutually supporting in shaping the experience.
\end{abstract}

\section{Author Keywords}

Visual markers; mobile interaction; museums; galleries; visitor interpretation

\section{ACM Classification Keywords}

H.5.m. Information interfaces and presentation (e.g., HCI): Miscellaneous;

\section{INTRODUCTION}

Visual markers, particularly QR codes, are widely adopted in museums and galleries as a way to exploit the capabilities of mobile devices carried by visitors to augment exhibits with digital content. An online survey conducted in 2013 by the Museum Association for the UK reported that $50 \%$ of those taking part had a mobile offer, with QR codes being the most popular technology deployed (63\%), followed by museum-provided audio-tours (46\%), mobileoptimised websites (45\%), and smartphone apps (39\%) [3].

Permission to make digital or hard copies of all or part of this work for personal or classroom use is granted without fee provided that copies are not made or distributed for profit or commercial advantage and that copies bear this notice and the full citation on the first page. Copyrights for components of this work owned by others than the author(s) must be honored. Abstracting with credit is permitted. To copy otherwise, or republish, to post on servers or to redistribute to lists, requires prior specific permission and/or a fee. Request permissions from Permissions@acm.org.
The appeal of QR codes is that they provide a low-cost way to deliver a broad range of applications. Simply by scanning a marker with their smartphone, visitors can get access to additional multimedia information about artefacts, play games such as treasure hunts and puzzles, and even contribute to the exhibition's interpretation by leaving comments and feedback. At the same time, visual markers are relatively cheap and easy to deploy, as no new technical infrastructure needs to be installed; visitors use their own devices to interact and only new labels need to be added.

However, visitor engagement with QR codes in museum settings can often be low. A study by Wein [34] found that QR codes were the least preferred mechanism by visitors for accessing background information on artworks with ease of use, enjoyability and distance identified as the main contributing factors. QR codes might not only be aesthetically unappealing, but also lead to a shift of attention away from the artwork [37]. Schultz [26] found that visitors at the Ryerson University Library and Museum of Inuit Art had an awareness of QR codes, but usage was observed to be low. The author suggests that a lack of perceived usefulness and misconceptions about ease of use might explain the low adoption. The study also reveals an assumption by staff and visitors that $\mathrm{QR}$ codes are only used for one-way provision of information, and highlights the opportunity of using the technology to initiate a conversation, as well as to personalize a visit to an institution.

In this paper we explore how visitors engage with a visual marker technology where the codes can be designed to be more aesthetic and meaningful, and can be created by the visitors themselves to contribute their own reflections to the exhibition. We chose Artcodes (previously known as Aesthticodes) $[2,21]$ as the approach for the authoring and recognition of aesthetically-pleasing patterns, as previous research has shown that people can easily understand the drawing rules and create interesting designs [21, 33].

The paper presents a study of the use of Artcodes to link audio recordings to photographic portraits exhibited in an art gallery. We also explore how visitors engaged with contributing their own reflections to the exhibition by drawing a marker and linking an audio comment. These hybrid artefacts were then displayed within the exhibition space. Our findings show that participants were positive about these kinds of markers as a means of providing 
additional information. They particularly appreciated the use of audio as media to augment their engagement with the portraits. However, we found that they became really enthusiastic when they were able to successfully draw their own code and link it to content. Subsequent visitors also really enjoyed viewing the codes left by previous visitors and interacting with this user contributed content. Our study revealed that visitors engaged with the aesthetic markers at the three levels of physical placement, aesthetic content and digital content. We suggest that these different levels need to be considered in the design of future visiting systems, which make use of such markers, to ensure they are mutually supporting in shaping the experience.

\section{USE OF VISUAL MARKERS IN MUSEUM SETTINGS}

There is a lot of interest by museums and cultural heritage sites in using mobile technologies to enhance visitor engagement [3]. Visual markers, usually in the form of QR codes, are most commonly used to provide additional information about exhibits, as this is a low-cost mechanism to overcome the space restrictions of text labels and enable the incorporation of multimedia (audio, video, animations) in an easily updatable form. Examples of this include the QR codes placed next to artworks in The Los Angeles County Museum of Art and Fort Wayne Museum of Art [4] to reveal biographical information about the artist, as well as QR codes linking to audio and video explanations of the objects on exhibit as shown in a early demonstration by the Museum in Urk, Holland [5].

QR codes can also be used to add more visitor interactivity through quizzes and treasure hunts. For example the Oslo Norsk Telemuseum enabled visitors to engage with the museum objects through solving a series of riddles and identifying the right answer by scanning the appropriate QR code [9]. Similarly a quiz and QR codes were used in an Art Gallery for students to identify correct paintings [22]. On the other hand, the exhibit on the 100th anniversary of the Boy Scouts in America across the Smithsonian National Museums in DC used QR codes to challenge visitors to a scavenger hunt to uncover links between scouting and some great national treasures [7]. Kuflik et al. describe the Treasure Hunt Game Generator System that was developed at the Hecht Museum to enable the museum staff to create new versions of treasure hunt games based on QR codes for special events, as well as for regular visits [20].

Going further, QR codes can also be used to enable visitors to contribute to exhibitions by sharing their own stories, interpretations, reflections and feedback. Although less explored, this application addresses recent museum concerns to support visitors in engaging with multiple interpretations, and creating, sharing and connecting with each other [39, 27]. The Tales of Things platform [29], which enables users to tag objects with stories through QR codes, has underpinned two notable examples. Workshops and events held at the National Museum of Scotland where visitors were encouraged to attach a $\mathrm{QR}$ code to an object and to 'record' a memory of using such an object [28] and the QRator project implemented at the Grant Museum of Zoology, which engaged visitors in meaning-making by asking them to respond to questions about different issues at exhibits [15]. Similarly, QR codes were installed to accompany objects at the Imperial War Museum with visitors being able to join the conversation by adding text comments to any item in the collection and reading what other people have to say [18].

However, while QR codes can be used to cost effectively support a range of relevant application in museums, visitor engagement with these markers is often low [26]. Previous research suggests that this might be because $\mathrm{QR}$ codes are aesthetically unappealing [37] and visitors are not aware of their usefulness [26]. Schultz also suggests that the most promising application of visual markers might be to personalize visits to institutions and to enable a conversation with visitors.

In this paper, we explore how visitors engage with handcrafted visual makers that can be designed to be more aesthetic and meaningful. We study both the use of such markers as labels for artefacts to provide complimentary information and as a mechanism for visitors to contribute to the exhibition themselves by creating physical as well as digital content. Our findings reveal (1) how museum visitors engaged with markers provided by the gallery versus those contributed by museum visitors, (2) provide detailed observation of how visitors interact with visual markers in galleries/museums (which is not detailed in previous literature) and (3) show what impact the use of more visually meaningful codes has on the experience.

\section{APPROACH}

We chose to augment the "Uncovering the Invisible" photography exhibition, which was due to be displayed at the Nottingham Lakeside Arts gallery. Created as a collaboration between British-Mexican photographers Pablo and Roxana Allison, it focuses on the diversity of backgrounds and life stories of the people that make up the Latin American community in the UK. Each portrait presents a photograph of a person and a label with a short description about them. In addition, audio recordings were available of the migrants featured in the photographs talking about their experiences of living in the UK. This presented us with an opportunity to explore the use of more aesthetic and meaningful visual markers as a mechanism for visitors to access the voice of each person featured on a portrait. Gallery staff and the photographers also saw the benefit of enabling the visitors to contribute to and grow the exhibition by sharing their own stories of life in the UK and reactions to the portraits. This enabled us to explore how visitors would engage with a visual marker technology, which allows them to design the visual appearance of the marker as well as the digital content it links to, and then how subsequent visitors interact with these additions to the exhibition. 
Implementing Interaction with Visual Markers

Recently, there has been growing interest in vision technologies that can recognize more aesthetic or natural images. Various approaches have been proposed to creating markers that contain the correct balance of features to make them recognizable by an image processing algorithm. These involve the designer selecting an existing image that fits requirements (e.g. Blippar [6], Vuforia [36]), modifying an image (e.g. ARTcodes [40], PiCode [17]) or drawing a new pattern following a set of rules (e.g. ARTTag [16], D-touch [13] and Artcodes [21]).

We chose to use Artcodes as the visual markers in our study as the drawing approach promotes visitor creativity and previous research has shown that the rules are easy to understand and follow, with a variety of interesting designs created [21, 33]. The technology involves recognizing topological structures in a live video, where a code is determined by the number of connected regions (which gives the number of digits in the code) and the number of blobs contained within each region (which gives the value of each digit). These are then written in ascending order (see Figure 1 for an example). The shapes of the regions and blobs, and ordering on the page are not considered, giving designers great flexibility to create varying designs. Thresholding to black and white is performed on the images before recognition, so color can be used in designs as long as there is sufficient contrast (as seen in Figure 2).

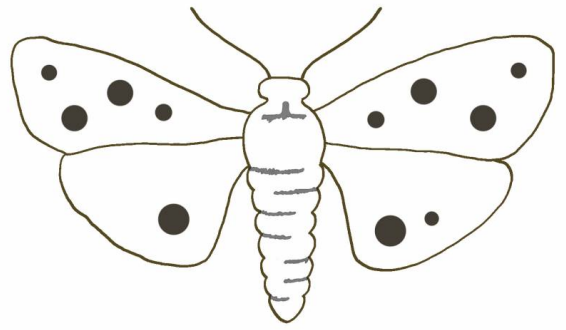

Figure 1. Artcode with 5 regions with the code 1:1:2:4:4

The "Uncovering the Invisible" exhibition consists of 22 portraits and we augmented 12 of them with Artcodes to trigger the associated audio recordings. The markers were created by a professional designer to represent the maps of the countries where the people came from. Figure 2 shows two examples and Figure 3 shows their placement.
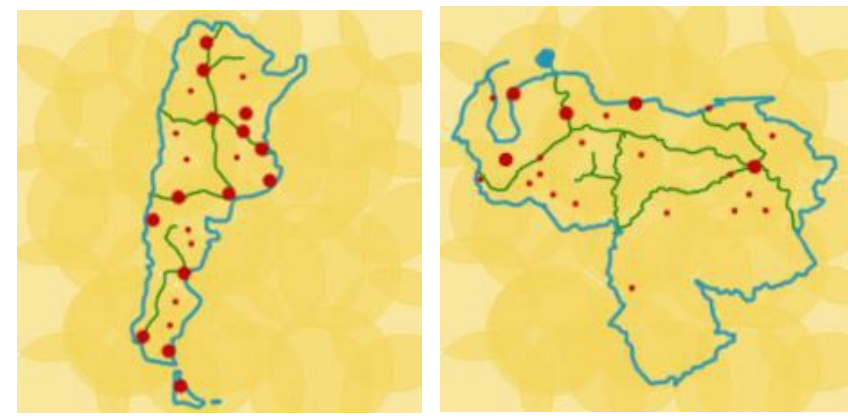

Figure 2. Artcodes representing the maps of Argentina and Venezuela

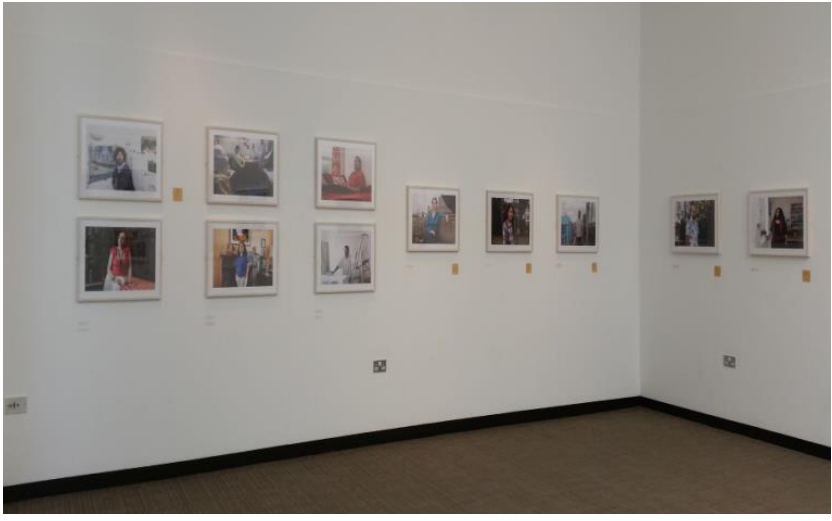

Figure 3. Placement of the markers in the gallery

We used the publicly available Artcodes app [2], which allows experiences to be created by specifying a set of codes and linking each one to a URL. We defined the "Uncovering the Invisible" experience and linked each of the 12 codes to the corresponding audio file on YouTube (where the voice recordings were already publicly available). By using the app and scanning an Artcode while browsing the exhibition, visitors could access the associated audio (see Figure 4).
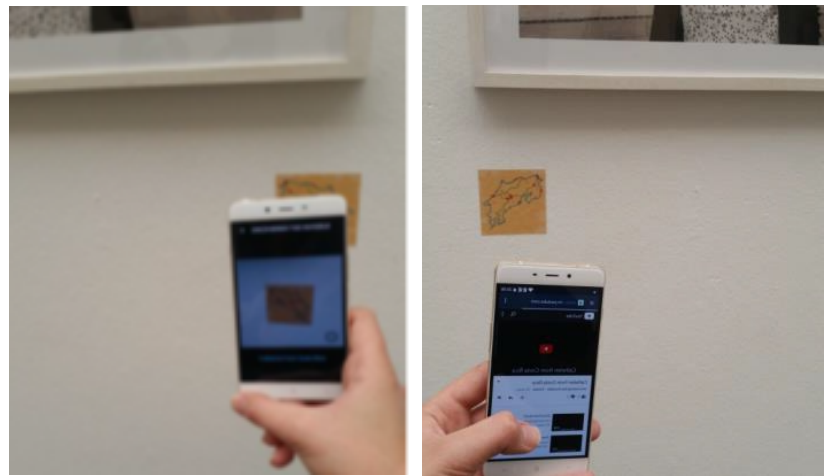

Figure 4. Scanning an Artcode label (left) and voice recording on YouTube (right)

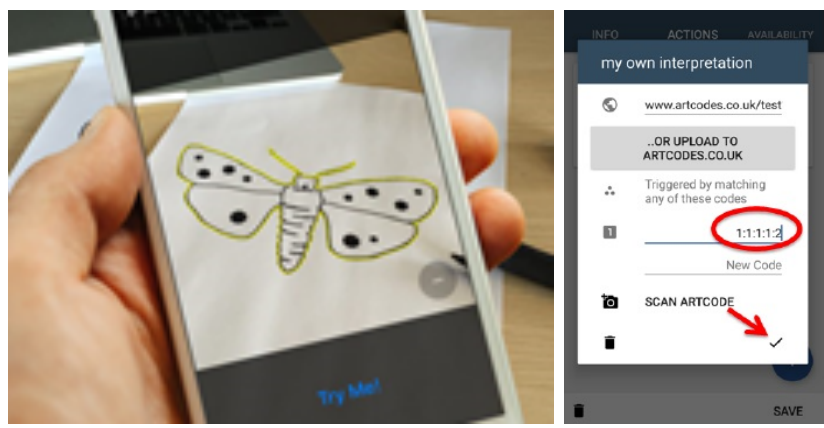

Figure 5. Testing an Artcode (left) and associating an audio file with a code (right)

The app also supported participants in drawing their own Artcode and linking this to digital content. To create their own drawing, which incorporates a valid code, visitors could use the Artcodes app functionality for testing (Figure 5 , left), which highlights the detected regions and displays the code. They could record their audio comment through 
an audio recording app on the smartphone and then upload it in the Artcodes app, associating it with an Artcode (Figure 5, right) either by manually writing the code or scanning the picture (which automatically detects and displays the code). The user could choose to share their contributions publicly or with selected people through SMS or social media (the audio file is uploaded on to a server in either case).

\section{Study Procedure}

\section{Participants}

Participants were recruited by advertising through the mailing lists and websites of the Nottingham Lakeside Arts gallery and the University of Nottingham Mexican society, as well as on physical notice boards around the University. 28 participants (11 male and 17 female) took part in the study. Of these 14 were aged (20-29), 11 (30-39), 2 (40-49) and 1 was over 60 years old. Participants came in groups of 2 or 3 friends apart from 4 who came alone. Only one of the participants was already familiar with the Artcodes technology.

\section{Study Structure}

The participants were split across 4 evaluation sessions each lasting approximately 2 hours. On arrival participants were briefly introduced to the exhibition, the aims of the study and the app (although they were not taught how to use it), asked to complete a consent form and provided with headphones. Then there were two distinct phases.

The first part (around 40-50 minutes) involved the visitors freely exploring the exhibition (with researchers available to offer assistance if needed), which after the first evaluation session also included contributions by visitors (Figure 6). This was followed by a focus group to discuss their experiences and views on augmenting exhibits with visual markers like Artcodes.

The second phase began with a demonstration of how to draw Artcodes and how to use the Artcodes app to create their own hybrid artefact comprising a visual code and audio recording. Participants were then provided with a worksheet to structure their creation process into the following sequence of activities: identify the aim of the artefact and who it is for (public, for specific people or private), plan the Artcode design and digital content and reflect on why they have been chosen, draw the code, record the audio, create the experience in the app and finally share it. This was followed by a focus group session to gather feedback on how participants felt about contributing to the exhibition in this way.

After we ran the first evaluation session, however, we found that participants spent a lot of time on drawing and experimenting with different Artcode designs. Consequently, even though they had all planned appropriate digital content (based on the worksheets), 11 out of 16 (that took part in the first evaluation session) only recorded audio as a means of testing that they had a working experience (e.g. "Hello" or "Testing"). We contacted these participants to ask them if they would like to send us the planned recording, so that it could be included in the exhibition and 7 of them provided this to us. For the remaining sessions, we swapped the order of drawing and recording audio, to check if this would make a difference and indeed, all subsequent participants made meaningful voice recordings. The hybrid artefacts were added to the exhibition space after each session for subsequent visitors to explore (see Figure 6). The choice of exhibiting all visitor contributions on one wall was made by the gallery staff who wanted to retain curatorial control of the presentation of the portraits.

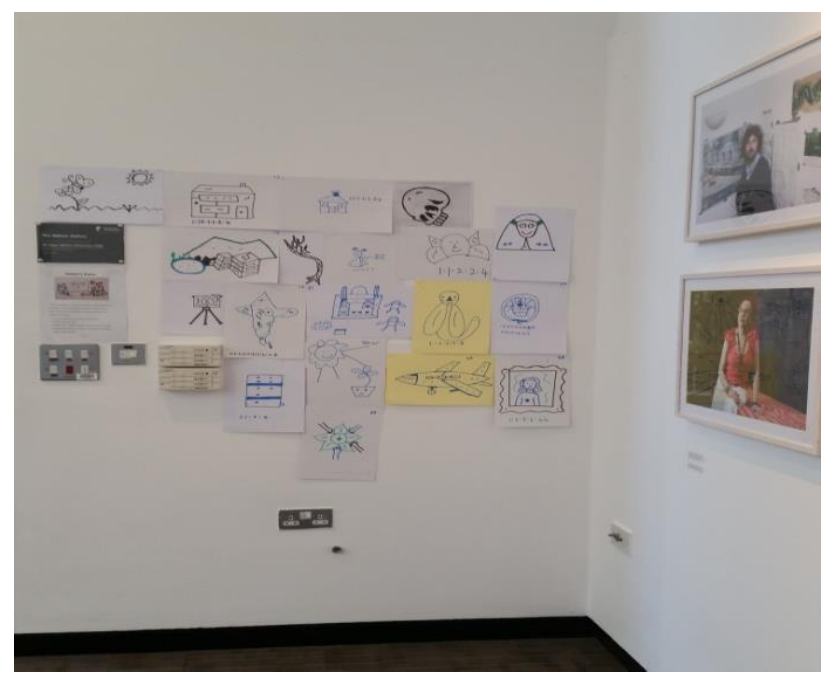

Figure 6. Exhibition of visitor contributed markers

\section{Data capture and analysis}

Data was collected through field notes, photographs, video recording of the visitors' interaction with the exhibition in phase 1 (a video camera was placed in a corner of the gallery), audio recording of the focus group sessions and the completed worksheets. The video recordings were qualitatively analyzed to understand how visitors interacted with the Artcodes accompanying the portraits and those created by previous participants, as well as with each other. The audio recordings of the focus group sessions were transcribed and thematically analyzed by two researchers through an active and reflexive process [8]. Data from the phase one focus group was used to complement observations with what participants' said about their interactions. Data from the phase two focus group, information provided in the worksheets and observations of this phase were combined to build a picture of what visitors created and why.

\section{FINDINGS}

Our analysis focused on the role of the markers in enhancing the visitors' experience of the exhibits. We were interested in how the visitors engaged with the markers, photographs and associated digital media. We were also interested in how the capabilities of the markers could be used to extend the experience beyond passive consumption 
to involve participants in a creative process to convey their reflections as part of the exhibition.

\section{Engagement with the Provided Exhibits and Markers}

Participants' experiences where broadly structured around three distinct phases. An initial engagement with the photograph and Artcode was followed by scanning the code and then a longer engagement with both the photograph and digital content.

After the initial brief engagement, all 28 participants scanned all 12 Artcode labels that had been provided. The scanning phase raised a number of issues that were often manifest in terms of the physical position of participants and markers. Uncertainty in code recognition led to users reconsidering the best position and distance to scan the image. For example, delays in recognition for two markers made some participants unsure about the best distance to scan from. They were observed stepping back and forth while scanning and in the focus group P9 (female, 20-29) reported:

"It seems that it will scan when focus like camera so you try to help camera to focus image so that is why."

Positioning was also significant in another way. The physical markers were placed in relation to the photographs being exhibited, with the photographs being the focus for visitor engagement rather than the markers. As a result markers that were placed lower could also be more challenging to scan in terms of finding an appropriate position to hold the phone (see Figure 7).

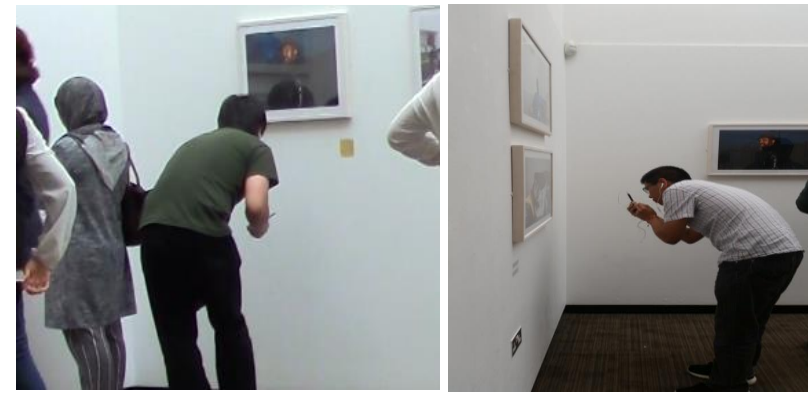

Figure 7. Participants trying to scan labels

Once participants had successfully scanned a marker and accessed the corresponding voice recording, they started a deeper engagement with the photograph and digital content. This was often marked by a change in orientation to the photograph and the marker, with the photograph often becoming the principle focus for participants. The majority of visitors (20 out of 28) moved back away from the marker to a position where they could still keep eye contact with the portrait (e.g. see Figure 8) and disengaged with the marker. This behavior was predominately observed in the first evaluation session when the largest number of participants (16) took part. In the focus group, visitors reported that they acted in this way because they were mindful of the presence of others and wanted to make space for them to interact while they were still engaged with the portrait, e.g. P1 (male, 30-39):

"You want to still keep eye contact with the person you are listening to but you do not need to distract other visitors. So you step back, you know, you still get this content to remember who you are listening to but to let other people get close and see the photo in more detail."

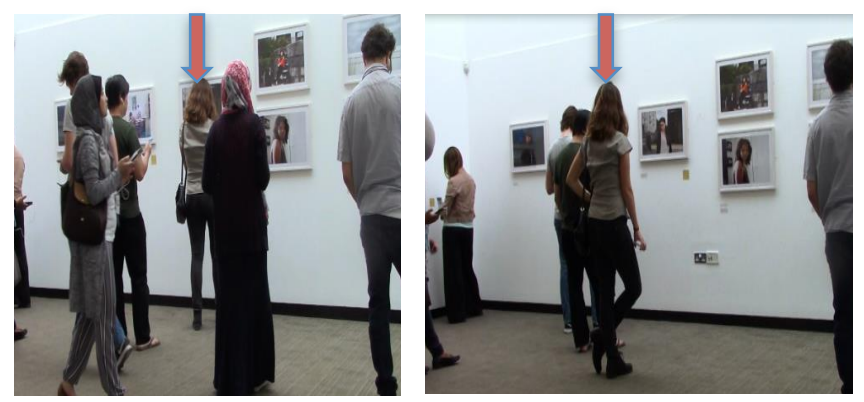

Figure 8. Scanning an Artcode from a close distance (left) and then moving back (right)

Few of the participants (6 out of 28) remained in place close to the portrait to listen to the audio after scanning. This was during the less busy sessions when there were not many other visitors around. The remaining 2 participants moved around the space after scanning while listening to the voice recording. They explained that they were looking for the next portrait to engage with.

The photograph was the dominant focus of engagement for participants. Engagement with the visual representation of the code tended to be determined by the extent to which participants recognized the image. The design of the markers was effective for those who were familiar with the shapes of Latin American countries and could recognize that the labels represented maps. In these cases the markers themselves conveyed an additional piece of information and this was appreciated. Some visitors, however, did not know what the pictures were meant to represent, e.g. P21 (male, 20-29):

"The people [who are] like good in geography ok, but for people like me, it was just like image not map."

\section{Engagement with the Provided Digital Content}

Audio was chosen as the media for the digital content to allow users to focus on the photographs in the exhibit. However, as participants moved from scanning the marker to a deeper engagement with the photograph, most participants looked at the phone screen when the voice recording started to play. They reported that this was to confirm that they had the right audio for the portrait they had selected.

We then observed that most participants (18) listened to the recording at the same time as they were looking at the portrait. They did this to observe the person in more detail, get a better understanding of them and feel more connected, e.g. P28 (female, 20-29) stated: 
"So I said I will enjoy the photos because that is what I am meant to look at. But also I think when you got a voice and you are looking at photo, you kind of make more of emotional link between them.",

In the focus groups there was general agreement that audio works well because it compliments the visual information from the portraits. It was suggested that text would be distracting and tiring to read. 4 from the 28 participants also thought that video would be an engaging format for the digital content, providing further information about the person depicted in the portrait. The others, however, strongly disagreed, arguing that it would take away from the engagement with the artworks, e.g. P27 (female, 20-29) reported:

"Video, for me is like losing the point because the art is there, so if you have a video or another picture in the app, maybe you going to look at that instead of the art."

On the other hand, the other (10) participants appeared more disengaged from the portraits as they were looking at the phone screen or other portraits rather than the one that they were listening to. They explained they were looking at the phone to check how long the audio is and then to focus on listening rather than attend to the portrait. This effect was amplified for some participants as the YouTube interface led them to expect a video instead of only audio.

\section{Design of the User Generated Hybrid Artefacts}

In general, participants wanted to design their hybrid artefact to express something personal about themselves or to add comments related to the exhibition. Most participants (18) focused on personal expression, with 15 planning their contribution to be about their own experience of living in the UK, or about their home country or other countries that they visited and 3 wanting to share their interests. On the other hand, 6 participants planned to comment on the exhibition and their experience of it.

The majority of participants (19) designed their artefact for the public, 8 for family and friends, who they hoped would visit the exhibition, and 1 participant had no target audience in mind. Half of those that focused on creating an artefact for a specific person (4 participants) specifically mentioned that their design would address the interests/preferences of the recipient.

\section{Crafting of Markers}

All participants were provided with the materials (paper and marker pens) to create Artcodes and were not restricted to specific codes or numbers of regions. Following instructions on how to draw (which were also provided as a printed sheet), all participants managed to create a valid marker within 15 minutes. A few of them drew more than one Artcode and some designed draft versions to learn how to draw valid patterns before designing the final marker.

Most Artcode designs (23) fitted the overall concept that participants had for their contribution as stated in the worksheets. For example, P17 (female, 20-29) designed a map of India (Figure 9, top left) to share her experience of leaving India and living in the UK to study. A picture of a house (Figure 9, top right), designed by P18 (female, over 60) was inspired by the exhibition and was used to leave a comment about the lives of people who come to the UK, while a skeleton (Figure 9, bottom left) designed by P11 (male, 30-39) was linked to information about Mexican illustrations. P23 (female, 40-49) created an airplane (Figure 9, bottom right) to symbolize travel but also because her son loves them, thus personalizing the illustration as her hybrid artefact was intended for a specific person - her son.

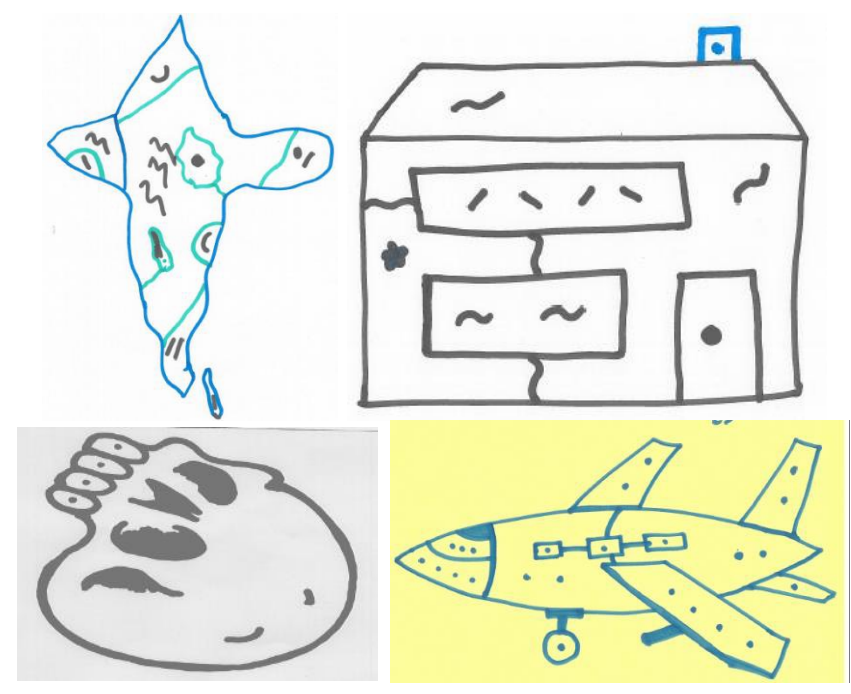

Figure 9. Example Artcodes created by participants

As mentioned in the previous section, 4 participants did not record a purpose for their artefact so it was not possible to make a judgement in those cases. The Artcode that did not appear to match was of a butterfly, designed by P8 (female, 30-39) (Figure10, left) whereas the stated purpose and recorded content were about life in the UK (without making a reference to the butterfly imagery).
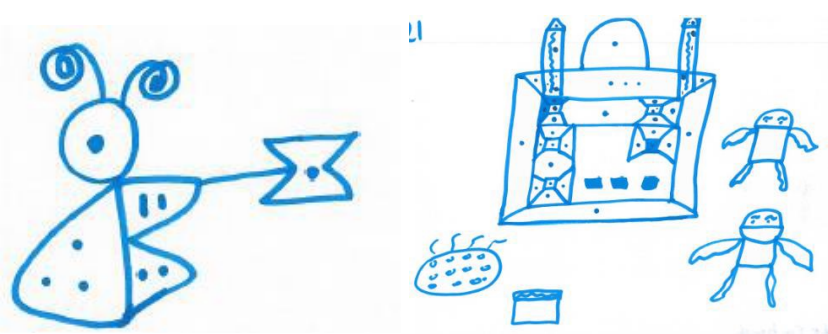

Figure 10. Marker not related to the content (left) and difficult to scan (right)

Only two participants struggled to get their Artcodes to scan and needed a lot of help from the workshop facilitators to make working versions. The technical issue with these designs was that there were a lot of blobs inside the regions, with most of them too close to the border to be recognized robustly by the app (e.g. Figure 10, right) designed by P21 (male, 20-29) showing a Hyderabad temple. P23 (female, 
40-49) was able to identify the issue with P21's Artcode that he struggled because he drew a complicated picture:

"It will be hard to start with complicated drawing but if you start with simple drawing, with the explanation that she gave it, will not be difficult".

\section{Recording of Digital Content}

All participants recorded their voice using a recording app on the smartphone. As discussed in the Study Structure section, during the first evaluation session 11 of the 16 participants did not initially record meaningful content. Although most provided content after the session when we invited them to send us the planned contribution, so that it could be included in the exhibition. During the subsequent 3 evaluation sessions, all participants recorded the intended digital content, resulting in 24 audio recordings in total. Their length ranged from 0:40 to 4:18 minutes.

The content of each audio recording either shared personal experiences associated with the themes of the exhibition or commented on the content of the exhibition. The former included reflections on life in the UK, the participant's home country and visiting other countries. For example, P7 who drew the house marker (Figure 9, top right) recorded a comment about family life in the UK:

"What I love about this country is a way they prioritize the family matters, so developing the work life and family. This one I love most in this country".

While another participant P15 (female, 30-39) designed a "teddy bear" Artcode to symbolize social relationships and reflected on her experience of this in her home country and in the UK:

"Nigeria is bubbly and lively place, the people there are full of energy, and passion and creativity and they show it. I have lived in the UK for sixteen years now. One major difference I would say that I find between people who live in the UK and Nigeria is that over here in the UK, people are a lot more conservative in terms of the social side...".

On the other hand others more directly responded to the exhibition with their contribution building upon the content of the exhibits. One example of this is the use of a skeleton marker (Figure 9, bottom left) by P11 where the audio comment recorded by the participant explains:

"Happy skeleton is about the Mexican tradition of drawing funny skeletons on the 2nd of November that is the Day of the Dead."

An example of the ways in which participants reflected on their experience of the exhibition itself is shown below by P28 (female, 20-29):

"Today I found through the use of Artcode app I was able to put a voice to the faces and that was really interesting to hear their experiences and created an emotional bond to the photos as you looked at them. You could gain more understanding of how people feel living here and what they experience being in this country. I also quite like the visitors' opinions of the art exhibition and their experiences in their country too... "

Participants also differed in how they began the audio recording with over half providing a brief biographical introduction to themselves and giving some background information, e.g. P8 (female, 30-39):

"Hi, I am X, a PhD student in computer science at the University of Y. I came from Bangladesh, a South Asian country..."

Whereas the others just provided a name or started to talk about their experiences straightaway, e.g. P21 (male, 2029):

\section{"Hi, I would like to talk about Hyderabad..."}

All participants in the first evaluation session (initially) recorded their voices in the workshop room, whereas in the other three sessions only 3 participants did so, with the others asking if they could go outside for this. Participants gave a number of reasons for this behavior. Some were worried about noise from other participants interfering with their audio recording. Others felt embarrassed to record their thoughts in the presence of other visitors, e.g. P28 (female, 20-29):

"I am shy to take phone calls in front of people so recording my voice to me is quite unnatural thing. I think if you are in a museum or something like that you can normally find a quiet corner to talk and then come back but $I$ don't think I would record my voice in a middle of a gallery."

This was particularly the case if they were not native speakers, as they wanted some privacy to prepare, e.g. P27 (female, 20-29):

"And I wanted to express what I have in my mind. And another issue of because English is my second language I think in Farsi and then translate what I have in Farsi..."

Despite these issues that some participants experienced with recording their voices, everyone highlighted that audio was the most suitable format to associate with the Artcode markers in museum and gallery settings. Participants felt that the audio medium compliments the visual information conveyed by the markers and enables them to communicate emotion in their reflections. It was suggested that providing a booth for audio recording in the museum or allowing visitors to complete the digital part of their artefact at home would address the privacy issues. There was agreement between all participants that they would prefer not to communicate their message through text. Video was suggested as an alternative, but some participants stated they would be too shy to make a video of themselves. It was proposed that providing the choice of audio or video recording might be a good solution. 


\section{Engagement with the Visitor Generated Artefacts}

Participants were highly engaged with the hybrid artefacts created by previous visitors (Figure 11). These were displayed within the gallery setting as part of the overall exhibition. In the three evaluation sessions where these were exhibited, we found that participants spent more time with them than interacting with the more formal Artcode labels provided with the portraits. In the focus group discussion they reported a number of reasons for this. All participants found the design of the visitors' markers to be meaningful and to relate to the digital audio content. They also felt a connection to these artefacts because they were created by visitors like them who had already experienced the exhibition and were curious to hear their experiences and reflections on the topic of the exhibition. The participants also appreciated points of view from different cultures and countries.

The visitors' markers also (unintentionally) turned out to be easier to interact with. The participants found the interface for playing the voice comments, which was directly integrated in the app, more convenient than the YouTube interface used for accessing the voices of the portraits. The visitors' markers were also more quickly recognized by the app due to their larger size and higher contrast. Unlike the labels, which were placed alongside exhibits, these markers were exhibits in their own right. Consequently, their position invited easy scanning. The codes replaced the photographs as the focal point for interaction. All visitors looked at the Artcode design while listening to the associated voice recording.

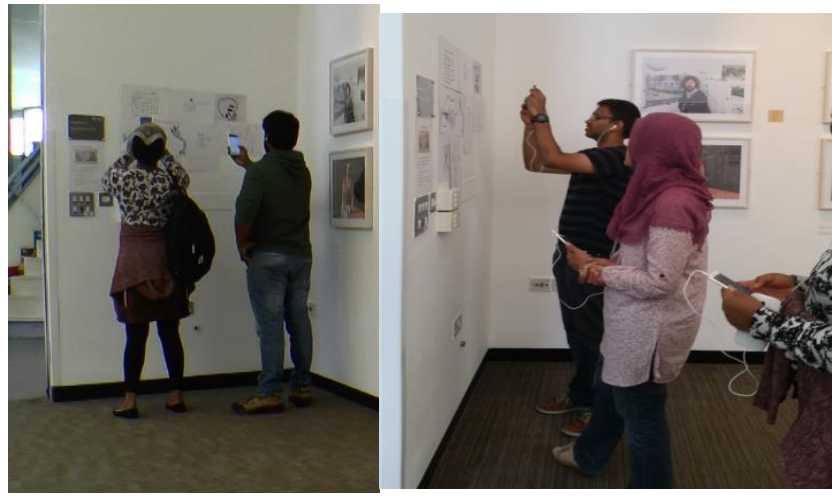

Figure 11. Participants interacting with the visitors' markers

As with the portrait markers, the behavior of scanning and moving back was also observed. This happened more frequently, usually when there was more than one other participant nearby, as the visitor markers were all placed on one wall close to each other and adjacent standing space was limited (see Figures 6 and 11).

\section{Social Interaction}

Although not designed for collaboration, the system enabled participants to engage in social interaction, with the overall experience being an inherently social activity. This was manifest in a number of ways during the visiting experience. People would observe others' interaction with the Artcodes and use the opportunity to help each other in scanning codes. The system was also appropriated to allow shared listening of the audio content. For example, a group of three friends visited the gallery together. This group stayed close to each other throughout the visit experience. They enthusiastically engaged with the Artcodes and the digital content. They approached the scanning of the codes collectively and at times they even shared the headphone earplugs to ensure they were listening to the recorded voices synchronously (Figure 12).

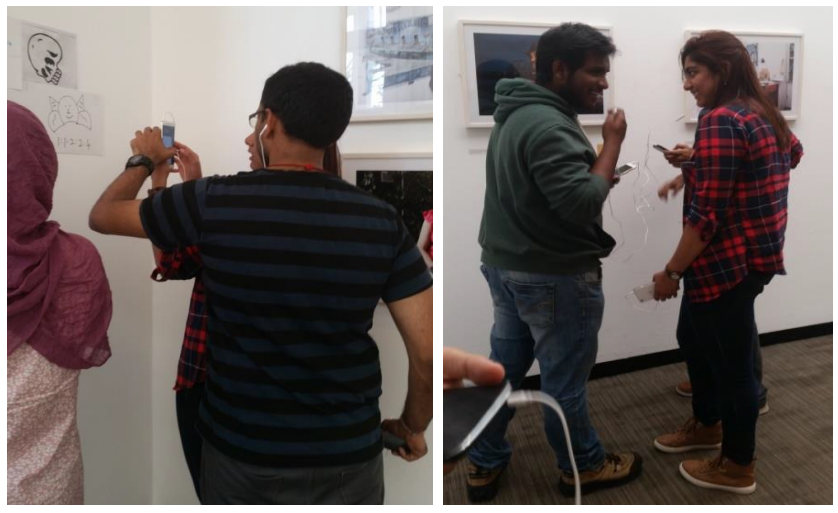

Figure. 12 Participants sharing the visiting experience

The process of designing the markers was also engaged with as a social activity promoting collaboration between visitors. Participants were actively engaged with the others' images during the drawing process and most of them (even those who came alone and did not know the others previously), talked, looked at other participants' designs, asked questions and helped each other (Figure 13). They reported that the activity was interesting and enjoyable, e.g. P19 (female, 20-29):

"It's nice like a puzzle and I really enjoy drawing things."

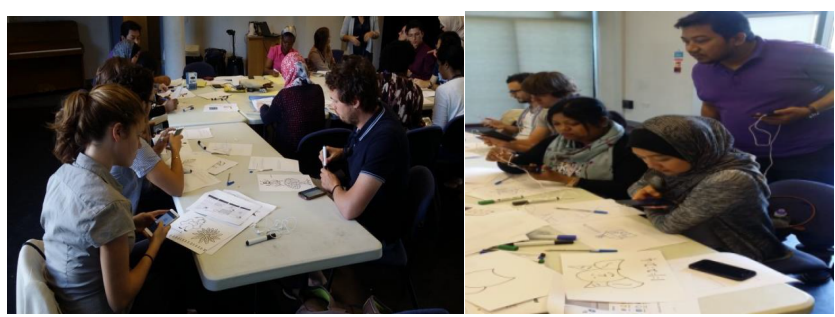

Figure 13. Participants engaged with drawing markers

\section{DISCUSSION}

Based on our study findings, we reflect on the lessons for using aesthetic meaningful markers as labels for exhibits and as a mechanism for visitors to craft a hybrid artefact that encapsulates a contribution to the exhibition.

\section{Augmenting Exhibits through Labels}

The placement of markers within the exhibition and their augmentation with audio commentary were viewed positively. We found that users successfully self-managed access to markers by being mindful of the presence of others and repositioning. This is in contrast to Wein [37] 
who suggests that visitors may be reluctant to engage with visual markers, such as $\mathrm{QR}$ codes, because they need to be able to get close to them in potentially crowded spaces. However, our study also highlights considerations for the use of this form of label as a technique for constructing visitors' experiences that exploit their mobile devices.

Our study revealed that visitors engaged with the aesthetic markers at three levels - physical placement, aesthetic content and digital content - that are mutually supportive in shaping the experience. Issues with any single level undermine the overall experience. This effect was notable in our study in terms of the challenges for some visitors arising from the positioning of labels and the legibility of the aesthetic content. It is also important that visitors can readily engage at multiple levels and shift their focus between these levels at low cost.

\section{Interaction with the Physical Placement of Markers}

Although it may seem an obvious point, placement needs to be considered carefully. It is critical to shaping the engagement across the other levels of interaction. Exhibitions are carefully curated and significant thought is given to the placement of artefacts in the space [39], and labels are often positioned such that they do not distract from the exhibit. One consequence of this is that labels might not be in a position that invites users to scan them easily. The ability to design markers that are aesthetically pleasing and complementary to the exhibits can potentially alleviate this problem by allowing such labels to be more prominent than QR codes. However, we still found that the positioning of labels would occasionally offer challenges for scanning. $\mathrm{Ng}$ and Shaikh also previously note issues with users having to scan labels from awkward positions when they were deployed in a botanical garden in Malaysia [23]. This arose from the placements of some of the labels, which were too far from the walkway. They suggest careful design of the physical labels, use of mounting stands to provide an optimum scanning distance for users, and realworld testing to reveal how real users will physically engage with the markers.

\section{Interaction with the Aesthetic Content of Markers}

Users also engaged at the level of the aesthetic content of markers. The images within the markers themselves can be used to add value to the experience of the exhibition. In our case they conveyed information about which country the person depicted on the portrait came from. However, the visual content needs to be designed with care to ensure that it is meaningful to users and supportive of the other levels. We found that a number of visitors were not able to interpret the images as country maps. It can be a challenge to strike a balance in the design of labels so that they convey additional information and/or imply their function, but are also sufficiently abstract to pique visitors' curiosity and encourage experimentation. The visual design of the label needs to be considered carefully in terms of its function in inviting visitors to scan it to engage with the digital content.

Interaction with the Digital Content.

The augmentation of this exhibition involved the assembly and linking of digital media hosted on a commodity service. Audio was presented to users through the interface provided by YouTube rather than by a dedicated functionality in the app. This form of construction is likely to be common for marker based mobile experiences. For example, there are a number of QR code based museum applications that link to Wikipedia [24]. However, this also introduces an interactional cost - that of understanding the change in interface, and switching back from the YouTube app or website to the Artcodes app - that makes it harder for visitors to shift their focus between the different levels involved. As we saw in our study, there is potential for confusion or distraction that arises through the presentation of digital media through a third party interface and there is a need for the careful assembly of the experience.

\section{Visitor Crafted Hybrid Artefacts}

As museums increasingly recognize the value of engaging visitors in a dialogue and enabling them to contribute their own interpretations [26, 36], a range of mechanisms to support visitor contributions have been explored. These include use of social media to encourage visitor engagement $[35,19,38]$, tagging of exhibits $[1,11,34]$ and specially developed interactive displays [12, 31]. Previous research projects, such as Retracing the Past [14] and Reminisce [10], have shown that allowing visitors to record voice messages is a particularly effective way of sharing memories and opinions with others in the museum setting.

We build on these mechanisms with our approach of visitors crafting hybrid artefacts that comprise both a voice recording and a hand drawn physical label. The ability to generate hybrid artefacts in reaction to the exhibition proved popular as a means of visitor expression and as an extension of the exhibition in its own right. It is interesting to note that all visitors chose to contribute new content (in effect creating a new exhibit), as outlined in the section Design of the User Generated Hybrid Artefacts, rather than offer reflections focusing on a specific portrait. Our results raise the possibility that hybrid contributions are more rewarding for the visitor than digital tags, which previously have been predominantly used to comment on existing exibits [11, 32]. In turn, participants who visited the gallery when the visitor-generated artefacts were displayed, found the hybrid contributions as engaging as the curated content.

However, unlike digital tags, it is important to note that hybrid contributions consume the museum's physical space. In our study, gallery space was kindly dedicated to participants to use, but for many museums space may be considered too valuable to spare for visitor contributions. Longer-term studies of hybrid artefacts as visitor contributions will need to consider how long the lifespan of a contribution should be, and strategies for rotating or 
retiring contributions to ensure that they do not overwhelm the museum.

\section{Supporting Multi-Level Crafting of Hybrid Artefacts}

The importance of the interplay between the multiple levels of interaction was evident as visitors recorded digital content, created aesthetic content by drawing labels and physically placed these in the exhibition in relation to other content. People expressed themselves through a combination of the physical image and the associated digital recording. As presented in the Findings section, most Artcode designs fitted the overall concept that participants had for their contribution, with the image linked in some way to the digital content.

The crafting of hybrid artefacts required effort and creativity and we suggest that consequently visitors put in more thought in the process, resulting in the generation of more interesting contributions than are usually found in an equivalent visitors' book or systems that allows objects to be tagged with text comments. Studies of maker communities have revealed that members value the pleasure of making and personal expression [30]. Allowing museum visitors to craft hybrid artefacts as a means of contributing to the museum can enable them to tap into these feelings. Our findings also contrast with those of Thom-Santelli at al's study [32], which found difference in engagement between expert and novice users in leaving digital tags. Arguably this lends further support for the value of drawing (and physical crafting more generally) in engaging visitors in making meaningful contributions.

However, the fact that the creation of hybrid artefacts requires a significant commitment from visitors also means that this process will require some form of scaffolding. It is important that support for crafting addresses the need to work at each of the three levels: people need to be supported in drawing the labels, linking the digital content and potentially placement in the space. We have seen from our study that dedicated sessions, facilitated by staff, work successfully. Alternatively, support for self-led creative activities could be provided in the form of a workstation with worksheets and instructions. However, the challenge of embedding sufficient contextual knowledge of the exhibit and how people relate to it, must not be underestimated.

Another direction for future research is to explore the placement of visitor contributions within the exhibition. In our study visitor drawings were displayed on one wall allocated by the gallery. These placements, worked well as all visitors chose to extend the exhibition with new drawings and voice comments rather than reflections focusing on a specific portrait. This was also the case for the 16 visitors in the first sessions, who at the point of creation, had not seen how their contributions would be displayed. In future research it would be interesting to explore if and how visitors can be allowed to be involved in the placement of their contribution within the exhibition and if this would affect what visitors create. This would further extend the role of visitors in shaping museum content but must be balanced against the need for museums to maintain overall curatorial control and moderation of content $[25,27]$.

\section{Scheduling within the Overall Visiting Experience}

The drawing of markers takes time, with participants experimenting with designs and then producing a final working version. It is important that this time commitment is designed for and managed as part of the overall visitor experience. It is important that this should reflect the creation of aesthetic and digital content and the physical placement in the setting. In our case, we found that the scheduling of marker drawing before the audio recording activity led participants to spend most of the available time on it, causing the remaining parts of the experience to be rushed, undermining the value of the hybrid artefact.

Finally, we would also suggest that people need space to generate images and audio commentary. It is worth noting that, although many museum visits are social, people often felt the need for privacy when recording audio content. Future work can also explore what the impact would be of allowing visitors to complete their hybrid artefacts at home.

\section{CONCLUSION}

Our study has shown that visual markers, such as Artcodes, which can be designed to convey visual meaning, can be combined with mobile devices to enhance the visitor experience of exhibits, especially when linked to complimentary audio content. Issues, such as the interface for accessing the digital media, appropriate marker design and marker placement, however, need to be considered carefully to ensure smooth interaction.

We have highlighted directions for future work on the use of visual markers, which can be crafted by the visitors, as a valuable mechanism for contributing new content to an exhibition. In our study the participants generated hybrid artefacts that were well thought-out and engaging for subsequent visitors, consisting of an image and audio comment. We raised the question of where these contributions might be embedded in the museum, how visitors can be involved in the process of placing their contributions in the museum, and what strategies for rotating and recycling contributions need to be implemented. We suggest that the drawing activity, which took time and effort, encouraged participants to reflect more deeply on the theme of the exhibition and focus on content that other visitors could relate to. To utilize visual markers in this way, however, requires further consideration of how, when and where to scaffold creative visitor activities.

\section{ACKNOWLEDGMENTS}

This work was supported by UK Engineering and Physical Sciences Research Council grant EP/M02315X/1 and by the EU Horizon 2020 grant 727040 (the GIFT project). 


\section{REFERENCES}

1. Ahern, S., Davis, M., Eckles, D., King, S., Naaman, M., Nair, R., ... \& Yang, J. 2006. Zonetag: Designing context-aware mobile media capture to increase participation. In Proceedings of the Pervasive Image Capture and Sharing, 8th Int. Conf. on Ubiquitous Computing, California, .

2. Artcodes website. Retrieved December 2017 from http://www.artcodes.co.uk/

3. Atkinson, R. 2013. Museum Practice: How are museums using mobile, from Museums Association. Retrieved December 2017 from https://www.museumsassociation.org/museumpractice/mobile-in-museums-2013/15102013-mobilesurvey-2013-results

4. BeQRious. Fort Wayne Art Museum Uses QR Codes To Educate Visitors. Retrieved December 2017 from http://beqrioustracker.com/fort-wayne-art-museumuses-qr-codes-to-educate-visitors/

5. BeQRious. Museum Urk First Dutch Museum to use New Media. Retrieved December 2017 from http://beqrioustracker.com/museum-urk-first-dutchmuseum-to-use-new-media/

6. Blippar website. Retrieved December 2017 from https://blippar.com/en/

7. Boy Scouts of America. Boy Scouts of America Takes Center Stage in Nation's Capital to Celebrate 100th Anniversary. Retrieved December 2017 from http://www.prnewswire.com/news-releases/boy-scoutsof-america-takes-center-stage-in-nations-capital-tocelebrate-100th-anniversary-99133979.html

8. Braun, V. and Clarke, V. 2006. Using thematic analysis in psychology. Qualitative Research in Psychology, 3 (2). pp. 77-101. ISSN 1478-0887

9. Ceipidor, U. B., Medaglia, C. M., Perrone, A., De Marsico, M., and Di Romano, G. 2009. A museum mobile game for children using $\mathrm{QR}$-codes. In Proceedings of the 8th International Conference on Interaction Design and Children (IDC '09), 282-283 http://dl.acm.org/citation.cfm?doid=1551788.1551857

10. Ciolfi, L. and McLoughlin, M. 2011. Challenges for the technological augmentation of open-air museums: Bridging buildings, artefacts and activities. Nordisk Museologi, (1), 15.

11. Cosley, D., Baxter, J., Lee, S., Alson, B., Nomura, S., Adams, P., Gay, G. 2009. A Tag in the Hand: Supporting Semantic, Social, and Spatial Navigation in Museums. In Proceedings of the 27th International Conference on Human Factors in Computing Systems (CHI'09), 1953-1962. http://doi.acm.org/10.1145/1518701.1518999

12. Cosley, D., Lewenstein, J., Herman, A., Holloway, J., Baxter, J., Nomura, S., ... \& Gay, G. 2008. ArtLinks: fostering social awareness and reflection in museums. In Proceedings of the SIGCHI Conference on Human Factors in Computing Systems (CHI '08), 403-412.

13. Costanza, E. Huang, J. 2009. Designable visual markers. In Proceedings of the SIGCHI conference on Human Factors in Computing Systems (CHI '09). http://dx.doi.org/10.1145/1518701.1518990

14. Ferris, K., Bannon, L., Ciolfi, L., Gallagher, P., Hall, T., \& Lennon, M. 2004. Shaping experiences in the hunt museum: a design case study. In Proceedings of the 5th conference on Designing interactive systems: processes, practices, methods, and techniques (pp. 205214). ACM.

15. Gray, S., Ross, C., Hudson-Smith, A., Warwick, C., and Terras, M. 2012. Enhancing Museum Narratives with the QRator Project: a Tasmanian devil, a Platypus and a Dead Man in a Box. In Proc. Museums and the Web (MW 2012).

16. Higashino, S., Nishi, S., \& Sakamoto, R. 2016. ARTTag: aesthetic fiducial markers based on circle pairs. In ACM SIGGRAPH 2016 Posters (p. 38). ACM.

17. Huang, W., \& Mow, W. H. 2013. PiCode: 2D barcode with embedded picture and ViCode: 3D barcode with embedded video. InProceedings of the 19th annual international conference on Mobile computing \& networking (pp. 139-142). ACM.

18. Imperial War Museum. Social Interpretation. Retrieved December 2017 from http://blogs.iwm.org.uk/social-interpretation/

19. Kidd, J. 2014. Museums in the New Mediascape: Transmedia, Participation. Ethics. New York: Routledge.

20. Kuflik, T., Rokeah, O., Salman, S. 2014. The Treasure Hunt Game Generator - a system and its application at the Hecht Museum. MW2014: Museums and the Web 2014. Available online: http://mw2014.museumsandtheweb.com/paper/thetreasure-hunt-game-generator-a-system-and-itsapplication-at-the-hecht-museum/

21. Meese, R., Ali, S., Thorne, E. C. et al. 2013. From codes to patterns: designing interactive decoration for tableware. In Proceedings of the SIGCHI Conference on Human Factors in Computing Systems (CHI '13), 931-940 http://dl.acm.org/citation.cfm?id=2466119

22. Mikalef, K. and Chorianopoulos, K. 2011. Game playing in the field: Effects of interactivity on learning and museum experience. In Proceedings of the DEG workshop on Involving End Users and Domain Experts in the Design of Educational Games.

23. Ng, K. H., \& Shaikh, S. P. 2016. Design of a mobile garden guide based on Artcodes. In User Science and Engineering (i-USEr), 2016 4th International Conference on (pp. 23-28). IEEE. 
24. Ross, Claire Bailey. 2012. What does success look like for museum QR code usage? Retrieved December 2017 from: https://claireyross.wordpress.com/tag/qr-codes/

25. Russo, A., Watkins, J., Kelly, L., \& Chan, S. 2008. Participatory communication with social media. Curator: The Museum Journal, 51(1), 21-31

26. Schultz, M. K. 2013. A case study on the appropriateness of using quick response $(\mathrm{QR})$ codes in libraries and museums. Library \& Information Science Research, 35(3), 207-215.

27. Simon, N. 2013. The Participatory Museum. Museums $2.0,2010$.

28. Speed, C and McDonald, J. 2013. TOTeM (Tales of Things and Electronic Memory). Retrieved December 2017 from: http://www.omnimuseum.org/id21.html

29. Tales of Things. Retrieved December 2017 from http://talesofthings.com

30. Tanenbaum, Joshua G., et al. 2013. "Democratizing technology: pleasure, utility and expressiveness in DIY and maker practice." Proceedings of the SIGCHI Conference on Human Factors in Computing Systems. ACM.

31. Taylor, N. 2014. Supporting community participation in interactive exhibits. In Proceedings of The International Symposium on Pervasive Displays ( $\mathrm{p}$. 74). ACM.

32. Thom-Santelli, J., Cosley, D., \& Gay, G. (2010). What do you know?: experts, novices and territoriality in collaborative systems. In Proceedings of the SIGCHI Conference on Human Factors in Computing Systems (CHI'10), 1685-1694. http://doi.org/10.1145/1753326.1753578
33. Thorn, E. C., Rennick-Egglestone, S., Koleva, B., Preston, W., Benford, S., Quinn, A., \& Mortier, R. 2016. Exploring large-scale interactive public illustrations. In Proceedings of the 2016 ACM Conference on Designing Interactive Systems (pp. 1727). ACM. https://doi.org/10.1145/2901790.2901826

34. Trant, J., \& Wyman, B. 2006. Investigating social tagging and folksonomy in art museums with steve. museum. In Collaborative Web Tagging Workshop at WWW2006, Edinburgh, Scotland.

35. Von Appen, K., Kennedy, B., \& Spadaccini, J. 2006. Community sites \& emerging sociable technologies. In Museums and the Web (pp. 197-206).

36. Vuforia website. Retrieved December 2017 from https:/www.vuforia.com/

37. Wein, L. 2014. Visual recognition in museum guide apps: do visitors want it? In Proceedings of the SIGCHI Conference on Human Factors in Computing Systems (CHI'14), 635-638. http://dl.acm.org/citation.cfm?doid=2556288.2557270

38. Weilenmann, A., Hillman, T., \& Jungselius, B. 2013. Instagram at the museum. In Proceedings of the SIGCHI Conference on Human Factors in Computing Systems (CHI '13), 1843. http://doi.org/10.1145/2470654.2466243

39. Whitehead, C. 2012. Interpreting art in museums and galleries. Routledge,

40. Zhe Yang, Yuting Bao, Chuhao Luo, et al. 2016. ARTcode: preserve art and code in any image. In Proceedings of the 2016 ACM International Joint Conference on Pervasive and Ubiquitous Computing (UbiComp '16), 904-915. http://dx.doi.org/10.1145/2971648.2971733 